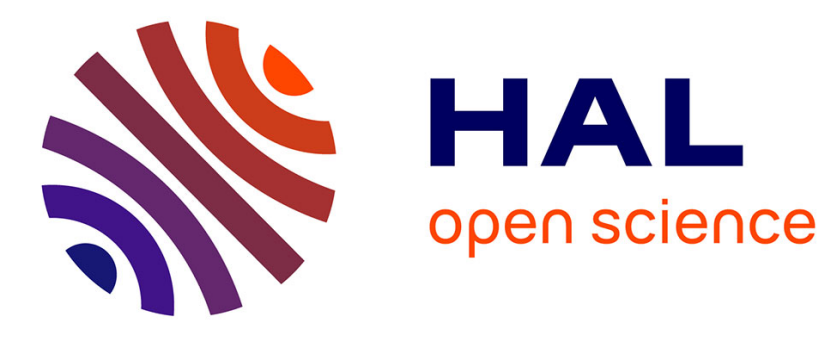

\title{
Typing control operators in the CPS hierarchy
}

Malgorzata Biernacka, Dariusz Biernacki, Sergueï Lenglet

\section{To cite this version:}

Malgorzata Biernacka, Dariusz Biernacki, Sergueï Lenglet. Typing control operators in the CPS hierarchy. PPDP - Principles and Practice of Declarative Programming, Jul 2011, Odense, Denmark. pp.149-160, 10.1145/2003476.2003498 . hal-00903834

\section{HAL Id: hal-00903834 \\ https://hal.inria.fr/hal-00903834}

Submitted on 13 Nov 2013

HAL is a multi-disciplinary open access archive for the deposit and dissemination of scientific research documents, whether they are published or not. The documents may come from teaching and research institutions in France or abroad, or from public or private research centers.
L'archive ouverte pluridisciplinaire HAL, est destinée au dépôt et à la diffusion de documents scientifiques de niveau recherche, publiés ou non, émanant des établissements d'enseignement et de recherche français ou étrangers, des laboratoires publics ou privés. 


\section{Typing Control Operators in the CPS Hierarchy}

\author{
Małgorzata Biernacka \\ Institute of Computer Science \\ University of Wrocław \\ mabi@cs.uni.wroc.pl
}

\author{
Dariusz Biernacki \\ Institute of Computer Science \\ University of Wrocław \\ dabi@cs.uni.wroc.pl
}

\author{
Sergueï Lenglet \\ Institute of Computer Science \\ University of Wrocław \\ serguei.lenglet@gmail.com
}

\begin{abstract}
The CPS hierarchy of Danvy and Filinski is a hierarchy of continuations that allows for expressing nested control effects characteristic of, e.g., nondeterministic programming or certain instances of normalization by evaluation. In this article, we present a comprehensive study of a typed version of the CPS hierarchy, where the typing discipline generalizes Danvy and Filinski's type system for control operators shift and reset. To this end, we define a typed family of control operators that give access to delimited continuations in the CPS hierarchy and that are slightly more flexible than Danvy and Filinski's family of control operators shift ${ }_{i}$ and reset $_{i}$, but, as we show, are equally expressive. For this type system, we prove subject reduction, soundness with respect to the CPS translation, and termination of evaluation. We also show that our results scale to a type system for even more flexible control operators expressible in the CPS hierarchy.
\end{abstract}

\section{INTRODUCTION}

In the recent years delimited continuations have been recognized as an important concept in the landscape of eager functional programming, with new practical [15, 16, 18, 19], theoretical $[1,2,4,13,20,24,26]$, and implementational [17, 21 advances in the field. Of the numerous control operators for delimited continuations, the static control operators shift and reset introduced by Danvy and Filinski in their seminal work [8] occupy a special position, primarily due to the fact that their definition has been based on the well-known concept of the Continuation-Passing Style (CPS) [23]. As such, shift and reset have solid semantic foundations [8, 10, $5]$, they are fundamentally related to other computational effects $[10,11]$ and their use is guided by CPS [5, 8]. A typical application of shift and reset, motivating their definition, are algorithms that nondeterministically generate elements of some collection, based on the success-failure continuation model of backtracking [8].

When iterated, the CPS translation leads to a hierarchy of continuations, generalizing the concept of the continuation and metacontinuation used to define the semantics of shift and reset. In terms of so defined CPS hierarchy, Danvy and Filinski proposed a hierarchy of control operators shift $i$ and reset $_{i}(i \geq 1)$ that generalize shift and reset, and that make it possible to separate computational effects that should exist independently in a program [8]. For example, in order to collect the solutions found by a backtracking algorithm implemented with shift ${ }_{1}$ and reset $_{1}$, one has to employ shift $_{2}$ and reset $_{2}$, so that there is no interference between searching and emitting the results of the search. The CPS hierarchy was also envisaged to account for nested computations in hierarchical structures. Indeed, as shown by the first two authors and Danvy [5], the hierarchy naturally accounts for normalization by evaluation algorithms for hierarchical languages of units and products, generalizing the problem of computing disjunctive or conjunctive normal forms in propositional logic.

So far, the CPS hierarchy has been studied mainly in the untyped setting. Danvy and Filinski defined it in terms of an untyped CPS translation and a valuation function of a denotational semantics [8], Danvy and Yang introduced an operational semantics for the hierarchy and built an SML implementation of the hierarchy based on this semantics [9], Kameyama presented an axiomatization for the hierarchy that is sound and complete with respect to the CPS translation [14], and Biernacka et al. derived abstract machines and reduction semantics for the hierarchy from the definitional evaluator [5].

A byproduct of Danvy and Yang's implementation in ML is a rather restrictive type system for the hierarchy, where at each level, the answer type of the continuation is fixed once and for all. This type system generalizes Filinski's type system for shift and reset [10], but it has not been investigated on a formal ground. To our knowledge, the only work on a formal type system for the hierarchy is Murthy's study [22], where the author proposes a more relaxed typing discipline allowing the delimited continuations of level $i$ to have various answer types, provided the answer type agrees with the type expected by the continuation at level $i+1$.

In this article, we go further and propose a type system that generalizes Danvy and Filinski's type system [7] which is the most expressive monomorphic type system for shift and reset. In Danvy and Filinski's type system, control effects can modify the answer type of the context (i.e., a first-order 
representation of the continuation) in which they occur, so statically, the answer type of the continuation at level $i$ can be different from the argument type of the continuation at level $i+1$. Furthermore, the control operators we consider are slightly more flexible than the original shift $i$ and $\operatorname{reset}_{i}$ family in that they capture the subsequent continuations to separate continuation variables and allow for throwing to tuples of continuations, where the continuations may come from different captures.

The overall goal of this article is to establish type-theoretic foundations of the CPS hierarchy and consequently, to shed more light on this beautiful but complex computational structure and, hopefully, inspire new theoretical and practical applications of the hierarchy. The contributions of this work can be summarized as follows:

- the definition of a new family of control operators in the CPS hierarchy that are slightly more flexible than the shift $_{i}$ and reset $_{i}$ family, given in terms of a CPS translation and reduction semantics provably sound with respect to the CPS translation (Sections 2.1 and $2.2)$;

- a type system à la Danvy and Filinski for the proposed operators, with proofs of subject reduction and soundness of the typing with respect to the CPS translation (Section 2.3);

- a proof of termination of evaluation in reduction semantics, using a context-based method of reducibility predicates (Section 2.4);

- a simulation of the presented operators with the original family of $\operatorname{shift}_{i}$ and $\operatorname{reset}_{i}$ (Section 2.5);

- a generalization of the presented results to a hierarchy of even more flexible control operators expressible in the CPS hierarchy (Section 3).

\section{FLEXIBLE CONTROL OPERATORS}

In this section, we present a hierarchy of flexible delimitedcontrol operators and we define a type system for it. We then show that it enjoys the standard correctness properties, such as subject reduction, soundness with respect to the CPS translation, and termination of evaluation. We also discuss the link between these operators and the first hierarchy of control operators due to Danvy and Filinski [8].

\subsection{Syntax}

The language of terms at an arbitrary level $n$ of the hierarchy extends the usual lambda terms with delimited-control operators capture $\mathcal{L}_{n}$, reset $\langle\cdot\rangle_{n}$, and throw $\hookleftarrow_{n}$, for $n \in \mathcal{N}_{+}$. At any level $n$, all operators inherited from lower levels $j<n$ are also available. In the following, we assume we have a set of term variables, ranged over by $x$, separate from $n$ pairwise distinct sets of continuation variables, ranged over by $k_{1}, \ldots, k_{n}$. The syntax of terms at level $n$ is defined as follows (where $1 \leq i \leq n)$ :

$$
\begin{aligned}
t::= & x|\lambda x . t| t t\left|\mathcal{L}_{i}\left(k_{1}, \ldots, k_{i}\right) . t\right|\langle t\rangle_{i} \mid \\
& \left(h_{1}, \ldots, h_{i}\right) \hookleftarrow_{i} t \\
h_{i}::= & k_{i} \mid E_{i} \\
v::= & \lambda x . t
\end{aligned}
$$

and the syntax of (call-by-value) evaluation contexts is given by (where $2 \leq i \leq n+1$ ):

$$
\begin{aligned}
E_{1} & ::=\bullet_{1}\left|v E_{1}\right| E_{1} t \mid\left(E_{1}, \ldots, E_{i}\right) \hookleftarrow_{i} E_{1} \\
E_{i} & ::=\bullet_{i} \mid E_{i} . E_{i-1}
\end{aligned}
$$

$\mathcal{L}_{i}\left(k_{1}, \ldots, k_{i}\right) . t$ are capture operators, each $\langle\cdot\rangle_{i}$ delimits the scope of the corresponding capture operator, and the throw constructs $\left(h_{1}, \ldots, h_{i}\right) \hookleftarrow_{i} t$ (similar to that of SML/NJ [12]) are used for applying a tuple of continuation variables or evaluation contexts to a term (or, throwing the term to the tuple).

In the original hierarchy of control operators [8], the shift construct $\mathcal{S}_{i} k . t$ is binding only one continuation variable instead of a tuple, and continuations are applied as regular functions, without any throw construct. Independently from this work, a throw construct $k \stackrel{\mathcal{S}}{\hookleftarrow}_{i} t$ can be introduced to distinguish continuation applications in the original hierarchy for typing purposes, as discussed in [4] (and in Section 2.3). Translating a term written with operators $\mathcal{S}_{i}$ and $\mathcal{S}_{i}$ to fit our system amounts to replacing singular variables $k$ by tuples of continuation variables of size $i$.

In a source program, a term can be thrown only to a tuple of continuation variables $\left(k_{1}, \ldots, k_{i}\right)$, and the programmer does not handle evaluation contexts explicitly. However, they can be introduced during evaluation, when some of the variables $k_{1}, \ldots, k_{i}$ are replaced by contexts captured by an operator $\mathcal{L}_{j}$. Therefore, we distinguish plain terms, i.e., terms that contain only throw constructs of the form $\left(k_{1}, \ldots, k_{i}\right) \hookleftarrow_{i} t$.

An abstraction $\lambda x$.t binds $x$ in $t$ and a capture construct $\mathcal{L}_{i}\left(k_{1}, \ldots, k_{i}\right) . t$ binds the variables $k_{1}, \ldots, k_{i}$ in $t$. The sets of free term and continuation variables are defined as usual, and we say a term is closed if it does not contain any free variables of any kind. A context is closed iff all terms occurring in it are closed. We equate terms up to $\alpha$-conversion of their bound variables.

Contexts $E_{i}$ can be seen as terms with a hole. We represent contexts inside-out, i.e., $\bullet_{1}$ represents the empty context of level $1, v E_{1}$ represents the "term with a hole" $E_{1}[v[]]$, $E_{1} t$ represents $E_{1}[[] t]$, and $\left(E_{1}, \ldots, E_{i}\right) \hookleftarrow_{i} E_{1}^{\prime}$ represents $E_{1}^{\prime}\left[\left(E_{1}, \ldots, E_{i}\right) \hookleftarrow_{i}[]\right]$. A context of level $i$ for $i=2, \ldots, n+$ 1 is a stack of contexts of level $i-1$ separated by a delimiter $\langle\cdot\rangle_{i-1}$. Therefore the empty context $\bullet_{i}$ of level $i$ stands for the term with a hole $\langle[]\rangle_{i-1}$, and $E_{i} \cdot E_{i-1}$ represents $E_{i}\left[\left\langle E_{i-1}[]\right\rangle_{i-1}\right]$. Formally, the function $\operatorname{plug}_{i}(1 \leq i \leq n+1)$ gives the term obtained by putting a term $t$ within a context $E_{i}$. We define $p l u g_{1}$ as follows:

$$
\begin{aligned}
\operatorname{plug}_{1}\left(t, \bullet_{1}\right) & =t \\
\operatorname{plug}_{1}\left(t, v E_{1}\right) & =\operatorname{plug}_{1}\left(v t, E_{1}\right) \\
\operatorname{plug}_{1}\left(t_{0}, E_{1} t_{1}\right) & =\operatorname{plug}_{1}\left(t_{0} t_{1}, E_{1}\right) \\
\operatorname{plug}_{1}\left(t,\left(E_{1}, \ldots, E_{i}\right) \hookleftarrow_{i} E_{1}^{\prime}\right) & = \\
& \operatorname{plug}_{1}\left(\left(E_{1}, \ldots, E_{i}\right) \hookleftarrow_{i} t, E_{1}^{\prime}\right)
\end{aligned}
$$




\section{CPS translation of terms}

$$
\begin{aligned}
\bar{x} & =\lambda k_{1} \ldots k_{n+1} \cdot k_{1} x k_{2} \ldots k_{n+1} \\
\overline{\lambda x \cdot t} & =\lambda k_{1} \ldots k_{n+1} \cdot k_{1}\left(\lambda x k_{1}^{\prime} k_{2}^{\prime} \ldots k_{n+1}^{\prime} \cdot \bar{t} k_{1}^{\prime} k_{2}^{\prime} \ldots k_{n+1}^{\prime}\right) k_{2} \ldots k_{n+1} \\
\overline{t_{0} t_{1}} & =\lambda k_{1} \ldots k_{n+1} \cdot \overline{t_{0}}\left(\lambda v_{0} k_{2}^{\prime} \ldots k_{n+1}^{\prime} \cdot \overline{t_{1}}\left(\lambda v_{1} k_{2}^{\prime \prime} \ldots k_{n+1}^{\prime \prime} . v_{0} v_{1} k_{1} k_{2}^{\prime \prime} \ldots k_{n+1}^{\prime \prime}\right) k_{2}^{\prime} \ldots k_{n+1}^{\prime}\right) \\
& k_{2} \ldots k_{n+1} \\
\overline{\langle t\rangle_{i}} & =\lambda k_{1} \ldots k_{n+1} \cdot \bar{t} \theta_{1} \ldots \theta_{i}\left(\lambda v_{0} k_{i+2}^{\prime} \ldots k_{n+1}^{\prime} \cdot k_{1} v_{0} k_{2} \ldots k_{i+1} k_{i+2}^{\prime} \ldots k_{n+1}^{\prime}\right) k_{i+2} \ldots k_{n+1} \\
\overline{\mathcal{L}_{i}\left(k_{1}^{\prime} \ldots k_{i}^{\prime}\right) \cdot t} & =\lambda k_{1} \ldots k_{n+1} \cdot \bar{t}\left\{k_{1} / k_{1}^{\prime}, \ldots, k_{i} / k_{i}^{\prime}\right\} \theta_{1} \ldots \theta_{i} k_{i+1} \ldots k_{n+1} \\
\overline{\left(h_{1} \ldots h_{i}\right) \hookleftarrow_{i} t}= & \lambda k_{1} \ldots k_{n+1} \cdot \bar{t}\left(\lambda v_{0} k_{2}^{\prime} \ldots k_{n+1}^{\prime} \cdot \llbracket h_{1} \rrbracket v_{0} \llbracket h_{2} \rrbracket \ldots \llbracket h_{i} \rrbracket\left(\lambda v_{1} k_{i+2}^{\prime \prime} \ldots k_{n+1}^{\prime \prime} \cdot k_{1} v_{1} k_{2}^{\prime} \ldots k_{i+1}^{\prime} k_{i+2}^{\prime \prime} \ldots k_{n+1}^{\prime \prime}\right)\right. \\
& \left.k_{i+2}^{\prime} \ldots k_{n+1}^{\prime}\right) k_{2} \ldots k_{n+1} \\
\text { where } \theta_{i}= & \quad \lambda x k_{i+1} \ldots k_{n+1} \cdot k_{i+1} x k_{i+2} \ldots k_{n+1} \quad \text { for } i=1, \ldots, n
\end{aligned}
$$

Refunctionalization of contexts

$$
\begin{aligned}
\llbracket k_{i} \rrbracket & =k_{i} \\
\llbracket \bullet_{i} \rrbracket & =\theta_{i} \\
\llbracket E_{1} t \rrbracket & =\lambda v_{0} k_{2} \ldots k_{n+1} \cdot \bar{t}\left(\lambda v_{1} k_{2}^{\prime} \ldots k_{n+1}^{\prime} \cdot v_{0} v_{1} \llbracket E_{1} \rrbracket k_{2}^{\prime} \ldots k_{n+1}^{\prime}\right) k_{2} \ldots k_{n+1} \\
\llbracket v_{0} E_{1} \rrbracket & =\lambda v_{1} k_{2} \ldots k_{n+1} \cdot v_{0}^{*} v_{1} \llbracket E_{1} \rrbracket k_{2} \ldots k_{n+1} \\
\llbracket\left(E_{1} \ldots E_{i}\right) \hookleftarrow_{i} E_{1}^{\prime} \rrbracket & =\lambda v_{0} k_{2} \ldots k_{n+1} \cdot \llbracket E_{1} \rrbracket v_{0} \llbracket E_{2} \rrbracket \ldots \llbracket E_{i} \rrbracket\left(\lambda v_{1} k_{i+2}^{\prime} \ldots k_{n+1}^{\prime} \cdot \llbracket E_{1}^{\prime} \rrbracket v_{1} k_{2} \ldots k_{i+1} k_{i+2}^{\prime} \ldots k_{n+1}^{\prime}\right) \\
\llbracket E_{i} \cdot E_{i-1} \rrbracket & =\lambda v k_{i+1} \ldots k_{n+1} \cdot \llbracket E_{i-1} \rrbracket v \llbracket E_{i} \rrbracket k_{i+1} \ldots k_{n+1} \\
\lambda x \cdot t^{*} & =\lambda x \cdot \bar{t}
\end{aligned}
$$

Figure 1: CPS translation

and for $i=2, \ldots, n+1$ we define:

$$
\begin{aligned}
\operatorname{plug}_{i}\left(t, \bullet_{i}\right) & =t \\
\operatorname{plug}_{i}\left(t, E_{i} . E_{i-1}\right) & =\operatorname{plug}_{i}\left(\left\langle p \operatorname{lug}_{i-1}\left(t, E_{i-1}\right)\right\rangle_{i-1}, E_{i}\right)
\end{aligned}
$$

We write $E_{i}[t]$ for the term $\operatorname{plug}_{i}\left(t, E_{i}\right)$.

In the following, we represent terms as programs in order to keep the layers of contexts delimited by the reset operators $\langle\cdot\rangle_{i}$ explicit. Such a representation is useful when writing reduction rules, where we have to decompose a term and locate its redex. It is also well suited for defining reducibility predicates to prove termination of well-typed terms (cf. Section 2.4).

Formally, a program at level $n$ of the hierarchy is defined as follows:

$$
p::=\left\langle t, E_{1}, \ldots, E_{n+1}\right\rangle .
$$

The program $\left\langle t, E_{1}, \ldots, E_{n+1}\right\rangle$ represents the term

$$
\operatorname{plug}_{n+1}\left(\left\langle\ldots\left\langle\operatorname{plug}_{2}\left(\left\langle p l u g_{1}\left(t, E_{1}\right)\right\rangle_{1}, E_{2}\right)\right\rangle_{2} \ldots\right\rangle_{n}, E_{n+1}\right),
$$

which can also be written as

$$
E_{n+1}\left[\left\langle\ldots\left\langle E_{2}\left[\left\langle E_{1}[t]\right\rangle_{1}\right]\right\rangle_{2} \ldots\right\rangle_{n}\right] .
$$

It can be seen from this definition that each term that we consider as a program at level $n$ is implicitly enclosed by the reset operators of each level from 1 to $n$. For example, the term $\lambda x . t$ will be considered as the program representing the term $\left\langle\ldots\langle\lambda x . t\rangle_{1} \ldots\right\rangle_{n}$.
To shorten the notation, we write plug $(p)$ for the term represented by the program $p$.

Since a term may have several decompositions, different programs can represent the same term. For example, for $n=2$, the program $\left\langle(\lambda x . t) v, \bullet_{1}, E_{2} . E_{1}, E_{3}\right\rangle$ may be also decomposed as $\left\langle\lambda x . t, \bullet_{1} v, E_{2} . E_{1}, E_{3}\right\rangle$, or as $\left\langle\langle(\lambda x . t) v\rangle_{1}, E_{1}, E_{2}, E_{3}\right\rangle$. We identify all decompositions of the same term by defining an equivalence relation on programs, as follows:

$$
p \sim p^{\prime}:=\operatorname{plug}(p)=\operatorname{plug}\left(p^{\prime}\right)
$$

and considering programs up to this equivalence.

\subsection{CPS Translation and Semantics}

We first define a CPS translation for our language, and then we derive the reduction semantics from it, using the same approach as Biernacka et al. [5]. The CPS translation, presented in Figure 1, extends the standard call-by-value CPS translation for the lambda calculus, and it uses the function $\llbracket \cdot \rrbracket$ which transforms contexts into continuations they represent (leaving continuation variables unchanged).

The call-by-value reduction semantics is shown in Figure 2. We write $t\{s / x\}$ for the usual capture-avoiding substitution of $s$ for the variable $x$ in $t$, and we write $t\left\{E_{1} / k_{1}\right\} \ldots\left\{E_{i} / k_{i}\right\}$ for the simultaneous capture-avoiding substitutions of contexts $E_{1}, \ldots, E_{i}$ for variables $k_{1}, \ldots, k_{i}$ in $t$. Terms $(\lambda x . t) v$ are the standard $\beta$-redexes of the call-by-value $\lambda$-calculus (the rule $\left.\left(\beta_{v}\right)\right)$. The reduction of a term $\mathcal{L}_{i}\left(k_{1}, \ldots, k_{i}\right) . t$ within contexts $E_{1}, \ldots, E_{n+1}$ consists in capturing the first 


$\begin{array}{lrrl}\left(\beta_{v}\right) & \left\langle(\lambda x . t) v, E_{1}, \ldots, E_{n+1}\right\rangle & \rightarrow_{\mathrm{v}} & \left\langle t\{v / x\}, E_{1}, \ldots, E_{n+1}\right\rangle \\ \left(\text { capture }_{i}\right) & \left\langle\mathcal{L}_{i}\left(k_{1}, \ldots, k_{i}\right) . t, E_{1}, \ldots, E_{n+1}\right\rangle & \rightarrow_{\mathrm{v}} & \left\langle t\left\{E_{1} / k_{1}, \ldots, E_{i} / k_{i}\right\}, \bullet_{1}, \ldots, \bullet_{i}, E_{i+1}, \ldots, E_{n+1}\right\rangle \\ \left(\text { reset }_{i}\right) & \left\langle\langle v\rangle_{i}, E_{1}, \ldots, E_{n+1}\right\rangle & \rightarrow_{\mathrm{v}} & \left\langle v, E_{1}, \ldots, E_{n+1}\right\rangle \\ \left(\text { throw }_{i}\right) & \left\langle\left(E_{1}{ }^{\prime}, \ldots, E_{i}^{\prime}\right) \hookleftarrow_{i} v, E_{1}, \ldots, E_{n+1}\right\rangle & \rightarrow_{\mathrm{v}} & \left\langle v, E_{1}{ }^{\prime}, \ldots, E_{i}^{\prime}, E_{i+1} \cdot\left(E_{i}, \ldots,\left(E_{2} . E_{1}\right), \ldots,\right), E_{i+2}, \ldots, E_{n+1}\right\rangle \\ & & & \\ & & & \text { for } 1 \leq i \leq n\end{array}$

Figure 2: Reduction rules

$i$ contexts $E_{1}, \ldots, E_{i}$ and substituting them for the variables $k_{1}, \ldots, k_{i}$ (the rule $\left(\right.$ capture $\left._{i}\right)$ ). When a value is thrown to a tuple of contexts $\left(E_{1}^{\prime}, \ldots, E_{i}^{\prime}\right)$ using a level- $i$ throw construct within contexts $E_{1}, \ldots, E_{n+1}$, then the new contexts $E_{1}^{\prime}, \ldots, E_{i}^{\prime}$ are reinstated as the current contexts, and the then-current contexts $E_{1}, \ldots, E_{i}$ are stacked on the context $E_{i+1}$ (the rule $\left(\right.$ throw $\left._{i}\right)$ ). Finally, if a value is surrounded by a reset of any level, then the delimiter is no longer needed and can be removed using the rule $\left(\right.$ reset $\left._{i}\right)$.

A redex is the first component (a term) of the program occurring on the left-hand side of each of the reduction rules above. A potential redex is either a proper redex or a stuck term, i.e., a term that neither is a value nor can be further reduced. The type system we propose in Section 2.3 ensures that a well-typed program cannot generate a stuck term in the course of its reduction. Because of the uniquedecomposition property of the calculus, the relation $\rightarrow_{\mathrm{v}}$ is deterministic.

Proposition 1 (UNIQUE-DECOMPOSITION PROPERTY). For all terms $t, t$ either is a value, or it decomposes uniquely into contexts $E_{1}, \ldots, E_{n+1}$ and a potential redex $r$, i.e., $t=$ plug $\left(\left\langle r, E_{1}, \ldots, E_{n+1}\right\rangle\right)$.

We define the evaluation relation as the reflexive and transitive closure of $\rightarrow_{\mathrm{v}}$. The result of the evaluation is a program value of the form $p_{v}:=\left\langle v, \bullet_{1}, \ldots, \bullet_{n+1}\right\rangle$. Hence, a program value is a term value surrounded by the (implicit) reset operators $\langle\cdot\rangle_{i}$ for $i=1, \ldots, n$.

Note that the semantics of the original shift $i$ can be retrieved by allowing only throws to tuples $E_{1}, \ldots, E_{i}$ captured by an operator $\mathcal{L}_{i}\left(k_{1}, \ldots, k_{i}\right)$.t, i.e., by forbidding throws to tuples built from different captures or to tuples of size $i$ built from a capture of size $j>i$.

\subsection{Type System}

We propose a type system for the CPS hierarchy. It is a conservative extension of the type system given by Biernacka and Biernacki for the first level of the hierarchy for shift and reset [4], which is itself a refinement of the classical type system of Danvy and Filinski [7]. The typing rules have been derived from the CPS image of the language (shown in Figure 1). We assume that we have a set of base type variables, ranged over by $b$. We let $S, T$ range over types for terms, and $C_{i}, D_{i}$ range over types for contexts $E_{i}$, for all $i=1, \ldots, n+1$. The syntax of types for terms and contexts is given below, where $1 \leq i \leq n$ :

$$
\begin{aligned}
S & ::=b \mid S \rightarrow\left[C_{1}, \ldots, C_{n+1}\right] \\
C_{n+1} & ::=\neg S \\
C_{i} & ::=S \triangleright C_{i+1} \ldots \triangleright C_{n+1}
\end{aligned}
$$

As in Danvy and Filinski's system, arrow types contain type annotations $S \rightarrow\left[C_{1}, \ldots, C_{n+1}\right]$ : a function with such a type can be applied to an argument of type $S$ within contexts of types $C_{1}, \ldots, C_{n+1}$. For $n=1$, the Danvy and Filinski's type $S_{U} \rightarrow{ }_{V} T$ corresponds to $S \rightarrow[T \triangleright U, \neg V]$.

In Danvy and Filinski's original type system, continuations are treated as regular functions; they are applied without any throw operator, and they are typed with regular (annotated) arrow types. As discussed in [4], this approach is too restrictive to type some interesting examples. In particular, it lacks context answer type polymorphism, which can be retrieved by representing captured continuations as contexts, and by using an explicit throw construct. Following this idea, we assign types to contexts $C_{i}$ that are not function types; a context of type $S \triangleright C_{i+1} \ldots \triangleright C_{n+1}$ can be plugged with a term of type $S$, and it can be put within contexts of types $C_{i+1}, \ldots, C_{n+1}$, respectively.

A type environment for term variables $\Gamma$ is a list of pairs $x: S$, and a type environment for continuation variables $\Delta$ is a list of pairs $k_{i}: C_{i}$. We derive typing judgments of the form $\Gamma ; \Delta \vdash_{n} t: C_{1}, \ldots, C_{n+1}$ for terms, and typing judgments of the form $\Gamma ; \Delta \vdash_{n} \quad E_{i}: C_{i}$ for contexts. If $\Gamma ; \Delta \vdash_{n} t:$ $C_{1}, \ldots, C_{n+1}$, then, under the assumptions $\Gamma, \Delta$, the term $t$ can be plugged into contexts of types $C_{1}, \ldots, C_{n+1}$. We do not need to mention explicitly the type of the term in the judgment, because we can retrieve it from the type $C_{1}$ of the first enclosing context, if needed. If $C_{1}=S \triangleright C_{2}^{\prime} \ldots \triangleright C_{n+1}^{\prime}$, then $t$ is of type $S$.

Danvy and Filinski's typing judgment $\Gamma ; \Delta|T \vdash t: S| U$ corresponds to the judgment $\Gamma ; \Delta \vdash_{1} t: S \triangleright T, \neg U$ in our system.

The typing rules are presented in Figure 3. We now briefly explain them and sketch how they were derived from the CPS translation of the language (of Figure 1).

The CPS defining equations are usually of the form $\overline{o p(t)}=$ $\lambda k_{1} \ldots k_{n+1} . \bar{t} k_{1}^{\prime} \ldots k_{n+1}^{\prime}$ for a given operator $o p$, and we want to generate a typing rule of the form

$$
\frac{\Gamma^{\prime} ; \Delta^{\prime} \vdash_{n} t: C_{1}^{\prime}, \ldots, C_{n+1}^{\prime}}{\Gamma ; \Delta \vdash_{n} \text { op }(t): C_{1}, \ldots, C_{n+1}} .
$$


Terms $(1 \leq i \leq n)$ :

$$
\begin{aligned}
& \overline{\Gamma, x: S ; \Delta \vdash_{n} x: S \triangleright C_{2} \ldots \triangleright C_{n+1}, C_{2}, \ldots, C_{n+1}} \quad \frac{1, \Delta \vdash_{n} \lambda x . t:\left(S \rightarrow\left[C_{1}^{\prime}, \ldots, C_{n+1}^{\prime}\right]\right) \triangleright C_{2} \ldots \triangleright C_{n+1}, C_{2}, \ldots, C_{n+1}}{\Gamma ; 1} \\
& \frac{\Gamma ; \Delta \vdash_{n} t_{0}:\left(S \rightarrow\left[C_{1}, \ldots, C_{n+1}\right]\right) \triangleright C_{2}^{\prime \prime} \ldots \triangleright C_{n+1}^{\prime \prime}, C_{2}^{\prime}, \ldots, C_{n+1}^{\prime} \quad \Gamma ; \Delta \vdash_{n} t_{1}: S \triangleright C_{2} \ldots \triangleright C_{n+1}, C_{2}^{\prime \prime}, \ldots, C_{n+1}^{\prime \prime}}{\Gamma ; \Delta \vdash_{n} t_{0} t_{1}: C_{1}, C_{2}^{\prime}, \ldots, C_{n+1}^{\prime}} \\
& \frac{\mathcal{I}_{1}\left(D_{1}\right) \quad \ldots \quad \mathcal{I}_{i}\left(D_{i}\right) \quad \Gamma ; \Delta \vdash_{n} t: D_{1}, \ldots, D_{i},\left(S \triangleright C_{i+2} \ldots \triangleright C_{n+1}\right), C_{i+2}^{\prime}, \ldots, C_{n+1}^{\prime}}{\Gamma ; \Delta \vdash_{n}\langle t\rangle_{i}: S \triangleright C_{2} \ldots \triangleright C_{n+1}, C_{2}, \ldots, C_{i+1}, C_{i+2}^{\prime}, \ldots, C_{n+1}^{\prime}} \\
& \frac{\mathcal{I}_{1}\left(D_{1}\right) \quad \ldots \quad \mathcal{I}_{i}\left(D_{i}\right) \quad \Gamma ; \Delta, k_{1}: C_{1}, \ldots, k_{i}: C_{i} \vdash_{n} t: D_{1}, \ldots, D_{i}, C_{i+1}, \ldots, C_{n+1}}{\Gamma ; \Delta \vdash_{n} \mathcal{L}_{i}\left(k_{1}, \ldots, k_{i}\right) . t: C_{1}, C_{2}, \ldots, C_{n+1}} \\
& C_{1}=S \triangleright C_{2} \ldots \triangleright C_{n+1} \quad C_{i+1}=T \triangleright C_{i+2}^{\prime} \ldots \triangleright C_{n+1}^{\prime} \\
& \Gamma ; \Delta \vdash_{n} h_{1}: C_{1} \quad \ldots \quad \Gamma ; \Delta \vdash_{n} h_{i}: C_{i} \\
& \frac{\Gamma ; \Delta \vdash_{n} t: S \triangleright C_{2}^{\prime \prime} \triangleright \ldots C_{i+1}^{\prime \prime} \triangleright C_{i+2} \triangleright \ldots C_{n+1}, D_{2}, \ldots, D_{n+1}}{{ }_{n}\left(h_{1}, \ldots, h_{i}\right) \hookleftarrow_{i} t: T \triangleright C_{2}^{\prime \prime} \triangleright \ldots C_{i+1}^{\prime \prime} \triangleright C_{i+2}^{\prime} \triangleright \ldots C_{n+1}^{\prime}, D_{2}, \ldots, D_{n+1}}
\end{aligned}
$$

\section{Contexts and continuation variables:}

$$
\begin{aligned}
& \frac{\mathcal{I}_{i}\left(C_{i}\right) \quad 1 \leq i \leq n+1}{\Gamma ; \Delta \vdash_{n} \bullet_{i}: C_{i}} \\
& \overline{\Gamma ; \Delta, k_{i}: C_{i} \vdash_{n} k_{i}: C_{i}} \\
& \frac{\Gamma ; \Delta \vdash_{n} E_{i}: C_{i} \quad \Gamma ; \Delta \vdash_{n} E_{i-1}: S \triangleright C_{i} \ldots \triangleright C_{n+1} \quad 2 \leq i \leq n+1}{\Gamma ; \Delta \vdash_{n} E_{i} . E_{i-1}: S \triangleright C_{i+1} \ldots \triangleright C_{n+1}} \\
& \frac{\Gamma ; \Delta \vdash_{n} E_{1}: C_{1} \quad \Gamma ; \Delta \vdash_{n} t_{1}: S \triangleright C_{2} \ldots \triangleright C_{n+1}, C_{2}^{\prime \prime}, \ldots, C_{n+1}^{\prime \prime}}{\Gamma ; \Delta \vdash_{n} E_{1} t:\left(S \rightarrow\left[C_{1}, \ldots, C_{n+1}\right]\right) \triangleright C_{2}^{\prime \prime} \ldots \triangleright C_{n+1}^{\prime \prime}} \\
& \frac{\Gamma ; \Delta \vdash_{n} v:\left(S \rightarrow\left[C_{1}, \ldots, C_{n+1}\right]\right) \triangleright C_{2}^{\prime \prime} \ldots \triangleright C_{n+1}^{\prime \prime}, C_{2}^{\prime \prime}, \ldots, C_{n+1}^{\prime \prime} \quad \Gamma ; \Delta \vdash_{n} E_{1}: C_{1}}{\Gamma ; \Delta \vdash_{n} v E_{1}: S \triangleright C_{2} \ldots \triangleright C_{n+1}} \\
& C_{1}=S \triangleright C_{2} \ldots \triangleright C_{n+1} \quad C_{i+1}=T \triangleright C_{i+2}^{\prime} \ldots \triangleright C_{n+1}^{\prime} \\
& \Gamma ; \Delta \vdash_{n} E_{1}: C_{1} \quad \ldots \quad \Gamma ; \Delta \vdash_{n} E_{i}: C_{i} \\
& \Gamma ; \Delta \vdash_{n} \quad E_{1}^{\prime}: T \triangleright C_{2}^{\prime \prime} \triangleright \ldots C_{i+1}^{\prime \prime} \triangleright C_{i+2}^{\prime} \triangleright \ldots C_{n+1}^{\prime} \\
& \overline{\Gamma ; \Delta \vdash_{n}\left(E_{1}, \ldots, E_{i}\right) \hookleftarrow_{i} E_{1}{ }^{\prime}: S \triangleright C_{2}^{\prime \prime} \triangleright \ldots C_{i+1}^{\prime \prime} \triangleright C_{i+2} \triangleright \ldots C_{n+1}}
\end{aligned}
$$

\section{Programs:}

$$
\frac{\Gamma ; \Delta \vdash_{n} E_{n+1}\left[\left\langle\ldots E_{1}[t] \ldots\right\rangle_{n}\right]: S \triangleright C_{2} \ldots \triangleright C_{n+1}, E_{2}, \ldots, E_{n+1}}{\Gamma ; \Delta \vdash_{n}\left\langle t, E_{1}, \ldots, E_{n+1}\right\rangle: S}
$$

Figure 3: A type system for the level $n$ of the CPS hierarchy

To this end, we annotate the CPS equation with the most liberal types, and we then deduce the types $C_{1}, \ldots, C_{n+1}$ from the types of $k_{1}, \ldots, k_{n+1}$, and $C_{1}^{\prime}, \ldots, C_{n+1}^{\prime}$ from the types of $k_{1}^{\prime}, \ldots, k_{n+1}^{\prime}$. For example, consider the CPS translation for term variables:

$$
\bar{x}=\lambda k_{1} \ldots k_{n+1} . k_{1} x k_{2} \ldots k_{n+1}
$$

The type of $k_{1}$ has to match those of $x, k_{2}, \ldots, k_{n+1}$ to make the application typable. No other constraints on types can be deduced for this equation, so we can derive the following typing rule for term variables:

$$
\overline{\Gamma, x: S ; \Delta \vdash_{n} x: S \triangleright C_{2} \ldots \triangleright C_{n+1}, C_{2}, \ldots, C_{n+1}}
$$

Consider now the CPS translation for reset:

$$
\overline{\langle t\rangle_{i}}=\lambda k_{1} \ldots k_{n+1} \cdot \bar{t} \theta_{1} \ldots \theta_{i} k_{i+1}^{\prime} k_{i+2} \ldots k_{n+1}
$$

with

$$
k_{i+1}^{\prime}=\left(\lambda v_{0} k_{i+2}^{\prime} \ldots k_{n+1}^{\prime} . k_{1} v_{0} k_{2} \ldots k_{i+1} k_{i+2}^{\prime} \ldots k_{n+1}^{\prime}\right)
$$

Assume that we type $k_{1}$ with $S \triangleright C_{2} \ldots \triangleright C_{n+1}$. To be able to type the continuation $k_{i+1}^{\prime}$ we have to assign types 
$C_{2}, \ldots, C_{n+1}$ to continuations $k_{2}, \ldots, k_{i+1}, k_{i+2}^{\prime}, \ldots, k_{n+1}^{\prime}$ and the type $S$ to $v_{0}$. Consequently, the continuation $k_{i+1}^{\prime}$ has type $S \triangleright C_{i+2} \ldots \triangleright C_{n+1}$. We do not have any constraints on the types of the continuation $k_{i+2}, \ldots, k_{n+1}$. Finally, we have to assign valid types $D_{1}, \ldots, D_{i}$ to the initial continuations $\theta_{1}, \ldots, \theta_{i}$. Let us recall the definition of the initial continuation:

$$
\theta_{j}=\lambda x k_{j+1} \ldots k_{n+1} \cdot k_{j+1} x k_{j+2} \ldots k_{n+1} .
$$

Therefore, a type $D_{j}=T \triangleright D_{j+1}^{\prime} \ldots \triangleright D_{n+1}^{\prime}$ is valid for $\theta_{j}$ iff $D_{j+1}^{\prime}=T \triangleright D_{j+2}^{\prime} \ldots \triangleright D_{n+1}^{\prime}$. We check this condition by defining a family of predicates $\mathcal{I}_{j}(1 \leq j \leq n)$ on context types as follows:

$$
\begin{aligned}
\mathcal{I}_{j}\left(C_{j}\right) & :=\exists S, C_{i+2}, \ldots, C_{n+1} . \\
C_{i} & =S \triangleright\left(S \triangleright C_{i+2} \ldots \triangleright C_{n+1}\right) \triangleright C_{i+2} \triangleright \ldots C_{n+1}
\end{aligned}
$$

and $\mathcal{I}_{n+1}\left(C_{n+1}\right)=$ True. We now have enough information to write the typing rule for $\langle\cdot\rangle_{i}$. Similarly, we derive typing rules for the remaining term constructors.

The typing rules for contexts can be derived by inspecting the equations defining the function $\llbracket \cdot \rrbracket$. For example, because $\llbracket \cdot \rrbracket$ translates $\bullet_{i}$ into the initial continuation $\theta_{i}$, the empty context of level $i$ can be typed with any type $C_{i}$ provided that $\mathcal{I}_{i}\left(C_{i}\right)$ holds. Note that we use the same typing judgment for continuation variables $\Gamma ; \Delta \vdash_{n} k_{i}: C_{i}$ as for contexts; it is just to make the typing rule for throw easier to write.

We point out that the typing rule for the original $\operatorname{shift}_{i} \mathcal{S}_{i}$, (and for reset $i$ and throw $\stackrel{\mathcal{S}}{\leftrightarrow}_{i}$, respectively) is the same as the rule for $\mathcal{L}_{i}$ (and for reset $_{i}$, throw $\hookleftarrow_{i}$, respectively).

Asai and Kameyama [2] defined a notion of pure term (i.e., a term free from control effects) in the polymorphic type system they designed for the level- 1 shift and reset. Using Danvy and Filinski's typing judgment, a typable term $t$ is pure iff we can derive $\Gamma ; \Delta|T \vdash t: S| T$ for any type $T$. We can generalize this notion to an arbitrary level $n$ of the CPS hierarchy. We see that for $i=n$ the typing rule for reset becomes

$$
\frac{\mathcal{I}_{1}\left(D_{1}\right) \quad \ldots \quad \mathcal{I}_{n}\left(D_{n}\right) \quad \Gamma ; \Delta \vdash_{n} t: D_{1}, \ldots, D_{n}, \neg S}{\Gamma ; \Delta \vdash_{n}\langle t\rangle_{i}: S \triangleright C_{2} \ldots \triangleright C_{n+1}, C_{2}, \ldots, C_{n+1}} .
$$

We notice that in the conclusion of this rule as well as in the conclusion of the rules for term variable and lambda abstraction, the types $E_{2}, \ldots, E_{n+1}$ are arbitrary, and the type of the first enclosing context is of the form $S \triangleright C_{2} \ldots \triangleright$ $C_{n+1}$. Therefore, we say that a typable term $t$ is pure iff we can derive $\Gamma ; \Delta \vdash_{n} t: S \triangleright C_{2} \ldots \triangleright C_{n+1}, C_{2}, \ldots, C_{n+1}$ for any $C_{2}, \ldots, C_{n+1}$.

We now state the main properties of the type system. First, we prove subject reduction: if a program $p$ is typable and reduces to $p^{\prime}$, then $p^{\prime}$ is typable with the same type. To this end, we will need to derive types for $t$ and $E_{1}, \ldots, E_{n+1}$ from the typing judgment $\Gamma ; \Delta \vdash_{n}\left\langle t, E_{1}, \ldots, E_{n+1}\right\rangle: S$. We will need a few lemmas:

LEMma 1. If $\Gamma ; \Delta \quad \vdash_{n} \quad E_{1}[t]: D_{1}, C_{2}, \ldots, C_{n+1}$ and $\mathcal{I}_{1}\left(D_{1}\right)$ hold, then $\Gamma ; \Delta \vdash_{n} E_{1}\left[\langle t\rangle_{1}\right]: C_{1}, C_{2}, \ldots, C_{n+1}$ and $\Gamma ; \Delta \vdash_{n} E_{1}: C_{1}$ hold for some $C_{1}$.
The proof is a straightforward structural induction on $E_{1}$.

Lemma 2. For $i=2, \ldots, n$, the following properties hold:

1. If

$$
\Gamma ; \Delta \vdash_{n} E_{i}\left[\langle t\rangle_{i-1}\right]: D_{1}, \ldots, D_{i}, C_{i+1}, \ldots, C_{n+1}
$$

and $\mathcal{I}_{j}\left(D_{j}\right)$ for all $j=1, \ldots, j$, then there exists $C_{i}$ such that $\Gamma ; \Delta \vdash_{n} \quad E_{i}: C_{i}$ and

$$
\Gamma ; \Delta \vdash_{n} t: D_{1}^{\prime}, \ldots, D_{i-1}^{\prime}, C_{i}, C_{i+1}, \ldots, C_{n+1}
$$

with $\mathcal{I}_{l}\left(D_{l}^{\prime}\right)$ for all $l=1, \ldots, i-1$.

2. If

$$
\begin{gathered}
\Gamma ; \Delta \vdash_{n} E_{i}\left[\langle t\rangle_{j}\right]: D_{1}, \ldots, D_{i}, C_{i+1}, \ldots, C_{n+1}, \\
\mathcal{I}_{l}\left(D_{l}\right) \text { holds for all } l=1, \ldots, i \text {, and } j \geq i, \text { then } \\
\Gamma ; \Delta \vdash_{n} t: D_{1}^{\prime}, \ldots, D_{j}^{\prime}, S \triangleright C_{j+2}^{\prime} \ldots \triangleright C_{n+1}^{\prime}, C_{j+2}, \ldots, C_{n+1} \\
\text { with } \mathcal{I}_{l}\left(D_{l}^{\prime}\right) \text { for all } l=1, \ldots, j \text { and } \\
\Gamma ; \Delta \vdash_{n} E_{i}: S \triangleright C_{i+1} \triangleright \ldots \triangleright C_{j+1} \triangleright C_{j+2}^{\prime} \triangleright \ldots \triangleright C_{n+1}^{\prime} .
\end{gathered}
$$

The main difficulty in proving subject reduction was to write down and prove Lemma 2 (and its counterpart for reconstruction, Lemma 6). The two properties stated in the lemma are proved simultaneously by induction on $i$ and on $E_{i}$.

\section{LEMMA 3. If}

$$
\begin{gathered}
\Gamma ; \Delta \vdash_{n} E_{n+1}\left[\langle t\rangle_{n}\right]: S \triangleright C_{2} \ldots \triangleright C_{n+1}, C_{2}, \ldots, C_{n+1} \\
\text { and } E_{n+1}=\bullet_{n+1} . E_{n}^{1}, \ldots, E_{n}^{m}, \text { then } \\
\Gamma ; \Delta \vdash_{n} t: D_{1}, \ldots, D_{n}, \neg T
\end{gathered}
$$

with $\mathcal{I}_{l}\left(D_{l}\right)$ for all $l=1, \ldots, n, \Gamma ; \Delta \vdash_{n} E_{n}^{1}: T^{\prime} \triangleright \neg S$, and $\Gamma ; \Delta \vdash_{n} E_{n+1}: \neg T$.

The proof is a straightforward structural induction on $E_{n+1}$. With these three lemmas, we can decompose a typed program as follows:

Lemma 4. If $\Gamma ; \Delta \vdash_{n}\left\langle t, E_{1}, \ldots, E_{n+1}\right\rangle: S$ and $E_{n+1}=$ $\bullet_{n+1} . E_{n}^{1} ., \ldots, E_{n}^{m}$, then there exist $C_{1}, \ldots, C_{n+1}$ such that $\Gamma ; \Delta \vdash_{n} \quad E_{i}: C_{i}$ for all $i=1, \ldots, n+1$, and $\Gamma ; \Delta \vdash_{n}$ $t: C_{1}, \ldots, C_{n+1}$. Furthermore, $\Gamma ; \Delta \vdash_{n} \quad E_{n}^{1}: T^{\prime} \triangleright \neg S$ is derivable for some $T^{\prime}$.

We now state auxiliary lemmas needed to perform the reverse operation: from a typed term $t$ and typed contexts $E_{1}, \ldots, E_{n+1}$, we want to deduce the type of the program $\left\langle t, E_{1}, \ldots, E_{n+1}\right\rangle$.

Lemma 5. If $\Gamma ; \Delta \vdash_{n} t: C_{1}, \ldots, C_{n+1}$ and $\Gamma ; \Delta \vdash_{n} E_{1}$ : $C_{1}$, then $\Gamma ; \Delta \vdash_{n} E_{1}[t]: D_{1}, C_{2}, \ldots, C_{n+1}$ is derivable and $\mathcal{I}_{1}\left(D_{1}\right)$ holds. 


$$
\begin{aligned}
& \mathcal{R}_{b}(v):=\text { True } \\
& \mathcal{R}_{S \rightarrow\left[C_{1}, \ldots, C_{n+1}\right]}\left(v_{0}\right):=\forall v_{1} \cdot \mathcal{R}_{S}\left(v_{1}\right) \rightarrow \forall E_{1} \cdot \mathcal{K}_{C_{1}}\left(E_{1}\right) \rightarrow \ldots \rightarrow \forall E_{n+1} \cdot \mathcal{K}_{C_{n+1}}\left(E_{n+1}\right) \rightarrow \mathcal{N}\left(\left\langle v_{0} v_{1}, E_{1}, \ldots, E_{n+1}\right\rangle\right) \\
& \mathcal{K}_{\neg S}\left(E_{n+1}\right):=\forall v \cdot \mathcal{R}_{S}(v) \rightarrow \mathcal{N}\left(\left\langle v, \bullet_{1}, \ldots, \bullet_{n}, E_{n+1}\right\rangle\right) \\
& \mathcal{K}_{S \triangleright C_{i+1} \ldots \triangleright C_{n+1}}\left(E_{i}\right):=\forall v \cdot \mathcal{R}_{S}(v) \rightarrow \forall E_{i+1} \cdot \mathcal{K}_{C_{i+1}}\left(E_{i+1}\right) \rightarrow \ldots \rightarrow \forall E_{n+1} \cdot \mathcal{K}_{C_{n+1}}\left(E_{n+1}\right) \\
& \mathcal{N}(p):=\exists v \cdot p \rightarrow_{\mathrm{v}}^{*}\left\langle v, \bullet_{1}, \ldots, \bullet_{n+1}\right\rangle \\
& \rightarrow \mathcal{N}\left(\left\langle v, \bullet_{1}, \ldots, \bullet_{i-1}, E_{i}, E_{i+1}, \ldots, E_{n+1}\right\rangle\right)
\end{aligned}
$$

Figure 4: Reducibility predicates

LEMma 6. The following properties hold:

1. If

$$
\Gamma ; \Delta \vdash_{n} t: D_{1}, \ldots, D_{i-1}, C_{i}, \ldots, C_{n+1},
$$

$\mathcal{I}_{l}\left(D_{l}\right)$ hold for all $l=1, \ldots, i-1$, and $\Gamma ; \Delta \vdash_{n} E_{i}: C_{i}$, then

$$
\Gamma ; \Delta \vdash_{n} E_{i}\left[\langle t\rangle_{i-1}\right]: D_{1}^{\prime}, \ldots, D_{i}^{\prime}, C_{i+1}, \ldots, C_{n+1}
$$

and $\mathcal{I}_{l}\left(D_{l}^{\prime}\right)$ hold for all $l=1, \ldots, i$.

2. If

$$
\begin{aligned}
& \Gamma ; \Delta \vdash_{n} t: D_{1}, \ldots, D_{j}, C_{j+1}, \ldots, C_{n+1}, \\
& \mathcal{I}_{l}\left(D_{l}\right) \text { hold for all } l=1, \ldots, j, C_{j+1}=S \triangleright C_{j+2}^{\prime} \ldots \triangleright \\
& C_{n+1}^{\prime} \text {, and } \\
& \Gamma ; \Delta \vdash_{n} E_{i}: S \triangleright C_{i+1} \triangleright \ldots \triangleright C_{j+1} \triangleright C_{j+2}^{\prime} \triangleright \ldots \triangleright C_{n+1}^{\prime}, \\
& \text { then } \\
& \quad \Gamma ; \Delta \vdash_{n} E_{i}\left[\langle t\rangle_{j}\right]: D_{1}^{\prime}, \ldots, D_{i}^{\prime}, C_{i+1}, \ldots, C_{n+1} \\
& \text { and } \mathcal{I}_{l}\left(D_{l}^{\prime}\right) \text { hold for all } l=1, \ldots, i \text {. }
\end{aligned}
$$

Lemma 7. If $\Gamma ; \Delta \vdash_{n} t: D_{1}, \ldots, D_{n}, \neg T, \mathcal{I}_{l}\left(D_{l}\right)$ hold for all $l=1, \ldots, n, E_{n+1}=\bullet_{n+1} . E_{n}^{1} ., \ldots, E_{n}^{m}, \Gamma ; \Delta \vdash_{n}$ $E_{n+1}: \neg T$, and $\Gamma ; \Delta \vdash_{n} E_{n}^{1}: T^{\prime} \triangleright \neg S$, then

$$
\Gamma ; \Delta \vdash_{n} E_{n+1}\left[\langle t\rangle_{n}\right]: S \triangleright C_{2} \ldots \triangleright C_{n+1}, C_{2}, \ldots, C_{n+1} .
$$

Lemma 8. If $\Gamma ; \Delta \vdash_{n} t: C_{1}, \ldots, C_{n+1}, \Gamma ; \Delta \vdash_{n} E_{i}: C_{i}$ for all $i=1, \ldots, n+1, E_{n+1}=\bullet_{n+1} \cdot E_{n}^{1}, \ldots, E_{n}^{m}$, and $\Gamma ; \Delta \vdash_{n} E_{n}^{1}: T^{\prime} \triangleright \neg S$, then $\Gamma ; \Delta \vdash_{n}\left\langle t, E_{1}, \ldots, E_{n+1}\right\rangle: S$ is derivable.

As usual, we need a substitution lemma to deal with the $\left(\beta_{v}\right)$ and $\left(\right.$ capture $\left._{i}\right)$ reduction rules.

\section{Lemma 9 (Substitution Lemma). The following hold:}

1. If $\Gamma, x: S ; \Delta \vdash_{n} t: C_{1}, \ldots, C_{n+1}$ and $\Gamma ; \Delta \vdash_{n} v$ : $S \triangleright C_{2}^{\prime} \ldots \triangleright C_{n+1}^{\prime}$, then $\Gamma ; \Delta \vdash_{n} t\{v / x\}: C_{1}, \ldots, C_{n+1}$.

2. If $\Gamma ; \Delta, K: D_{1} \triangleright \ldots \triangleright D_{i} \vdash_{n} t: C_{1}, \ldots, C_{n+1}$ and $\Gamma ; \Delta \vdash_{n} \quad E_{j}: D_{j}$ for all $j \in 1, \ldots, i$, then $\Gamma ; \Delta \vdash_{n}$ $t\left\{\left(E_{1}, \ldots, E_{i}\right) / K\right\}: C_{1}, \ldots, C_{n+1}$.
Using these lemmas, we can prove subject reduction.

Theorem 1 (Subject Reduction). If $\Gamma ; \Delta \vdash_{n} p: S$ and $p \rightarrow_{\mathrm{v}} p^{\prime}$ then $\Gamma ; \Delta \vdash_{n} p^{\prime}: S$.

We now state the correctness of the type system with respect to the CPS translation. To this end, we first introduce a translation of the types of terms and contexts into simple types as follows:

$$
\begin{aligned}
\bar{b} & =b \\
\overline{S \rightarrow\left[C_{1} \ldots C_{n+1}\right]} & =\bar{S} \rightarrow \overline{C_{1}} \rightarrow \ldots \rightarrow \overline{C_{n+1}} \rightarrow o \\
\overline{C_{i}} & =\overline{S \triangleright C_{i+1} \ldots \triangleright C_{n+1}} \\
& =\bar{S} \rightarrow \overline{C_{i+1}} \rightarrow \ldots \rightarrow \overline{C_{n+1}} \rightarrow o \\
\overline{C_{n+1}} & =\overline{\neg S}=\bar{S} \rightarrow o
\end{aligned}
$$

where $o$ is an abstract answer type.

We also define a translation on typing contexts in the usual way, i.e., $\bar{\Gamma}$ (resp., $\bar{\Delta})$ is obtained from $\Gamma($ resp., $\Delta)$ by translating all types occurring in $\Gamma$ (resp., $\Delta$ ).

Proposition 2 (Soundness of TyPing WrT. CPS). The following implications ensure the soundness of the typing of the hierarchy with respect to the CPS translation, where $\vdash$ denotes the standard typing judgments deriving simple types for pure lambda terms:

1. If $\Gamma ; \Delta \vdash_{n} t: C_{1}, \ldots, C_{n+1}$, then $\bar{\Gamma} ; \bar{\Delta} \vdash \bar{t}: \overline{C_{1}} \rightarrow$ $\ldots \rightarrow \overline{C_{n+1}} \rightarrow o$.

2. If $\Gamma ; \Delta \vdash_{n} \quad E_{i}: C_{i}$, then $\bar{\Gamma} ; \bar{\Delta} \vdash \llbracket E_{i} \rrbracket: \overline{C_{i}}$, for all $i=1, \ldots, n$.

We can show that reductions in the hierarchy are sound with respect to the CPS translation:

Proposition 3 (Soundness of Reduction wrt. CPS). If $p \rightarrow_{\mathrm{v}} p^{\prime}$, then $\bar{p}={ }_{\beta \eta} \overline{p^{\prime}}$.

Proposition 3 is proved using a characterization of the CPSimage of a program in terms of its CPS-translated components: 
Proposition 4 (Characterization of CPS image). If $p=\left\langle t, E_{1}, \ldots, E_{n}\right\rangle$, then

$$
\bar{p}={ }_{\beta \eta} \lambda k_{1} \ldots k_{n+1} \cdot \bar{t} \llbracket E_{1} \rrbracket \ldots \llbracket E_{n} \rrbracket\left(\lambda v_{0} . k_{1} v_{0} k_{2} \ldots k_{n+1}\right) .
$$

Proposition 4 states that the CPS translation of a program is convertible to the CPS term obtained first, by CPS translating the term component $t$ of the program, then applying it to continuations obtained by refunctionalizing the successive contexts $E_{i}$, and finally applying it to a continuation of the highest level that collects all the current continuations $k_{i}$ (as a refunctionalized stack of lower-level contexts/continuations).

\subsection{Termination of Evaluation}

We prove termination for call-by-value evaluation, extending the method used by Biernacka and Biernacki [4] for level-1 shift and reset to the level $n$ of the hierarchy. The proof technique is a context-based variant of Tait's reducibility predicates [25]. For simplicity, we restrict ourselves to closed terms, but the result can be extended to open terms.

We define mutually inductive families of predicates on terms and contexts as shown in Figure 4 . The predicate $\mathcal{R}_{S}$, indexed by term types, is defined on values, and the predicates $\mathcal{K}_{C_{i}}$, indexed by context types, are defined on evaluation contexts for all $i=1, \ldots, n+1$. A value of a function type is reducible iff the program obtained by applying this value to a reducible value and put within reducible contexts normalizes (i.e., it evaluates to a program value). In turn, a context $E_{i}$ of level $i$ is reducible iff the program $\left\langle v, \bullet_{1}, \ldots, \bullet_{i-1}, E_{i}, E_{i+1}, \ldots, E_{n+1}\right\rangle$ built from any reducible value $v$ and any reducible contexts $E_{i+1}, \ldots, E_{n+1}$ of the appropriate types normalizes. The predicate $\mathcal{N}$ is defined on closed programs: $\mathcal{N}(p)$ holds iff $p$ evaluates to a program value in the call-by-value strategy (the strategy is enforced by the grammar of contexts $E_{1}$ ).

In the following, for any closed value $v$ we write $\vdash v: S$ iff there exist $C_{2}, \ldots, C_{n+1}$ such that $\cdot ; \cdot \vdash_{n} v: S \triangleright C_{2} \ldots \triangleright$ $C_{n+1}, C_{2}, \ldots, C_{n+1}$. Because $v$ is pure, we do not care about the specific $C_{2}, \ldots, C_{n+1}$, as discussed in Section 2.3.

In order to prove termination, we need the following two lemmas.

Lemma 10. If $\mathcal{I}_{i}\left(C_{i}\right)$, then $\mathcal{K}_{C_{i}}\left(\bullet_{i}\right)$.

Lemma 11. Let $t$ be a plain term such that $\Gamma ; \Delta \vdash_{n} t$ : $C_{1}, \ldots, C_{n+1}$, where $\Gamma=x_{1}: T_{1}, \ldots, x_{n}: T_{n}$ and $\Delta=$ $k_{i_{1}}^{1}: D_{i_{1}}^{1}, \ldots, k_{i_{m}}^{m}: D_{1}^{m}$. Let $\vec{v}$ be closed values such that $\vdash v_{i}: T_{i}$ and $\mathcal{R}_{T_{i}}\left(v_{i}\right)$ for all $i=1, \ldots, n$. Let $\vec{E}_{i}$ be closed contexts such that $; \cdot \vdash_{n} \quad E_{i_{j}}^{j}: D_{i_{j}}^{j}$ and $\mathcal{K}_{D_{i_{j}}^{j}}\left(E_{i_{j}}^{j}\right)$. Let $E_{1}^{\prime}, \ldots, E_{n+1}^{\prime}$ be closed contexts such that $\cdot ; \cdot \vdash_{n} \quad E_{i}^{\prime}: C_{i}$ and $\mathcal{K}_{C_{i}}\left(E_{i}^{\prime}\right)$. Then $\mathcal{N}\left(\left\langle t\left\{\vec{v} / \vec{x}, \vec{E}_{i} / \overrightarrow{k_{i}}\right\}, E_{1}^{\prime}, \ldots, E_{n+1}^{\prime}\right\rangle\right)$ holds.

The proof of Lemma 11 is similar to the one of the analogous lemma in [4]; this lemma is used to prove the following result:
Theorem 2 (Termination of evaluation). Let $t$ be a closed plain term such that $; \cdot \cdot \vdash_{n} t: C_{1}, \ldots, C_{n+1}$, and $\mathcal{I}_{i}\left(C_{i}\right)$ hold for all $i=1, \ldots, n+1$. Then $\mathcal{N}\left(\left\langle t, \bullet_{1}, \ldots, \bullet_{n+1}\right\rangle\right)$ holds.

Theorem 2 is stated for plain terms only, since it is only for such terms that we are able to control the reducibility property of captured contexts occurring in them (here, it can only happen by substituting a reducible context for a continuation variable).

\subsection{Expressiveness}

In this section, we prove that the hierarchy of operators $\mathcal{L}_{i}$ and $\leftarrow_{i}$ is as expressive as the hierarchy of the original operators shift $\mathcal{S}_{i}$ and throw $\stackrel{\mathcal{S}}{\leftarrow}_{i}$ [8]. We also consider an alternative throw operator $\stackrel{\mathcal{C}}{\leftarrow}$ i and compare it with $\hookleftarrow_{i}$.

Regular shift and throw operators. Because the original hierarchy of shift $_{i}$ and reset $i$ operators with the addition of a throw $_{i}$ operator can be embedded in our hierarchy, the typing rules and the associated results carry over to the original hierarchy.

We now show how to express $\mathcal{L}_{i}$ and $\hookleftarrow_{i}$ with the regular shift and throw. We define a translation $(\cdot)^{\circ}$ which rewrites terms with $\mathcal{L}_{i}$ and $\hookleftarrow_{i}$ into terms with $\mathcal{S}_{i}$ and $\stackrel{\mathcal{S}}{\hookleftarrow}_{i}$ in the following way:

$$
\begin{aligned}
& x^{\circ}=x \\
& (\lambda x . t)^{\circ}=\lambda x \cdot t^{\circ} \\
& \left(t_{0} t_{1}\right)^{\circ}=t_{0}^{\circ} t_{1}^{\circ} \\
& \langle t\rangle_{i}^{\circ}=\left\langle t^{\circ}\right\rangle_{i} \\
& \left(\mathcal{L}_{i}\left(k_{1}, \ldots, k_{i}\right) \cdot t\right)^{\circ}=\mathcal{S}_{1} k_{1}^{\circ} \cdot \mathcal{S}_{2} k_{2}^{\circ} \ldots \mathcal{S}_{i} k_{i}^{\circ} \cdot t^{\circ} \\
& \left(\left(h_{1}, \ldots, h_{i}\right) \hookleftarrow_{i} t\right)^{\circ}= \\
& \left(\lambda x .\left\langle h_{i}^{\circ} \stackrel{\mathcal{S}}{\hookleftarrow}_{i} \ldots\left\langle h_{2}^{\circ} \stackrel{\mathcal{S}}{\hookleftarrow}_{2}\left\langle h_{1}^{\circ} \stackrel{\mathcal{S}}{\hookleftarrow}_{1} x\right\rangle_{1}\right\rangle_{2} \ldots\right\rangle_{i}\right) t^{\circ} \\
& E_{i}^{\circ}=\left(\bullet_{1}, \bullet_{2}, \ldots, \bullet_{i-1}, E_{i}\right) \\
& k_{i}^{\circ}=\left(k_{1}^{i}, \ldots, k_{i}^{i}\right)
\end{aligned}
$$

We assume that the translation of continuation variables $k_{i}^{\circ}$ is deterministic (i.e., it generates always the same tuple of variables, written $\left.\left(k_{i}^{\circ}(1), \ldots, k_{i}^{\circ}(i)\right)\right)$ and that the translation of two different variables generates disjoint tuples. The idea of the translation is to perform successive shifts, in order to capture tuples of contexts of the form $\left(\bullet_{1}, \bullet_{2}, \ldots, \bullet_{i-1}, E_{i}\right)$. In the translation of $\leftarrow_{i}$, the contexts $E_{i}$ are then restored successively by throwing to these tuples. Note that in the translated terms, we always throw to a tuple of contexts captured by a singular shift, therefore we respect the semantics of $\mathcal{S}_{i}$.

In order to prove the soundness of the translation with respect to CPS, we define a function $\widehat{\imath}$, which returns the CPS translation of $h_{i}^{\circ}$.

$$
\begin{aligned}
\widehat{E_{i}^{\circ}} & =\llbracket E_{i} \rrbracket \\
\widehat{k_{i}^{\circ}} & =\lambda x k_{i+1} \ldots k_{n+1} . k_{i}^{\circ}(1) x k_{i}^{\circ}(2) \ldots k_{i}^{\circ}(i) k_{i+1} \ldots k_{n+1}
\end{aligned}
$$

In the following, we write $t\left\{\left(k_{1}^{\prime}, \ldots, k_{i}^{\prime}\right) / k_{i}^{\circ}\right\}$ as a shorthand for $t\left\{k_{1}^{\prime} / k_{i}^{\circ}(1), \ldots, k_{i}^{\prime} / k_{i}^{\circ}(i)\right\}$. 
LEMma 12. The following equalities hold for all $1 \leq j \leq$

$$
\begin{aligned}
& \overline{\mathcal{S}_{j} k_{j}^{\circ} \ldots \mathcal{S}_{i} k_{i}^{\circ} \cdot t}=_{\beta \eta} \quad \lambda k_{1} \ldots k_{n+1} . \\
& \bar{t}\left\{\left(\theta_{1} \ldots \theta_{i-1}, k_{i}\right) / k_{i}^{\circ}, \ldots,\left(k_{1} \ldots k_{j}\right) / k_{j}^{\circ}\right\} \\
& \theta_{1} \ldots \theta_{i} k_{i+1} \ldots k_{n+1} \\
& \overline{\left\langle h_{i}^{\circ} \stackrel{\mathcal{S}}{\hookleftarrow}_{j} \ldots\left\langle h_{1}^{\circ} \stackrel{\mathcal{S}}{\hookleftarrow}_{1} x\right\rangle_{1}\right\rangle_{j}}={ }_{\beta \eta} \quad \lambda k_{1} \ldots k_{n+1} . \widehat{h_{1}^{\circ}} x \widehat{h_{2}^{\circ}} \ldots \widehat{h_{j}^{\circ}} \\
& \left(\lambda v_{0} k_{j+2}^{\prime} \ldots k_{n+1}^{\prime} . k_{1} v_{0} k_{2} \ldots k_{j+1} k_{j+2}^{\prime} \ldots k_{n+1}^{\prime}\right) \\
& k_{j+2} \ldots k_{n+1} \\
& \widehat{k_{i}^{\circ}}\left\{\left(\theta_{1} \ldots \theta_{i-1}, k_{i}^{\prime}\right) / k_{i}^{\circ}\right\} \quad=_{\beta \eta} \quad k_{i}^{\prime}
\end{aligned}
$$

Using this lemma, we can prove the simulation theorem below.

THEOREM 3. Let $t$ be a term and let $k_{i_{1}}^{1}, \ldots, k_{i_{j}}^{j}$ (written with $\mathcal{L}_{i}$ and $\hookleftarrow_{i}$ ) be its free continuation variables. Then $\overline{t^{\circ}}={ }_{\beta \eta} \bar{t}\left\{\widehat{k_{i_{1}}^{10}} / k_{i_{1}}^{1}, \ldots, \widehat{k_{i_{j}}^{j} \circ} / k_{i_{j}}^{j}\right\}$.

In particular, for closed terms we have $\overline{t^{\circ}}={ }_{\beta \eta} \bar{t}$.

An alternative throw operator. In some cases we may want to consider an alternative throw operator $\stackrel{\mathcal{C}}{\leftarrow}_{i}$, which restores saved contexts and discards the current ones without storing them. Formally, its CPS translation is defined as follows:

$$
\begin{aligned}
& \overline{\left(h_{1}, \ldots, h_{i}\right) \stackrel{\mathcal{C}}{\hookleftarrow}_{i} t}=\lambda k_{1} \ldots k_{n+1} . \\
& \bar{t}\left(\lambda v_{0} k_{2}^{\prime} \ldots k_{n+1}^{\prime} \cdot \llbracket h_{1} \rrbracket v_{0} \llbracket h_{2} \rrbracket \ldots \llbracket h_{i} \rrbracket k_{i+1}^{\prime} \ldots k_{n+1}^{\prime}\right) \\
& k_{2} \ldots k_{n+1}
\end{aligned}
$$

and the corresponding reduction rule is:

$$
\begin{aligned}
&\left\langle\left(E_{1}^{\prime}, \ldots, E_{i}^{\prime}\right) \stackrel{\mathcal{C}}{\hookleftarrow}_{i} v, E_{1}, \ldots, E_{n+1}\right\rangle \rightarrow_{\mathrm{v}} \\
&\left\langle v, E_{1}^{\prime}, \ldots, E_{i}^{\prime}, E_{i+1}, \ldots, E_{n+1}\right\rangle
\end{aligned}
$$

The operators $\stackrel{\mathcal{C}}{\leftarrow}_{i}$ and $\hookleftarrow_{i}$ can be defined one in terms of the other as follows:

$$
\begin{aligned}
\left(h_{1}, \ldots, h_{i}\right) \stackrel{\mathcal{C}}{\hookleftarrow}_{i} t= & \left(\lambda x \cdot \mathcal{L}_{i}\left(k_{1}^{\prime}, \ldots, k_{i}^{\prime}\right) \cdot\left(h_{1}, \ldots, h_{i}\right) \hookleftarrow_{i} x\right) t \\
& \text { where }\left\{k_{1}^{\prime}, \ldots, k_{i}^{\prime}\right\} \cap\left\{h_{1}, \ldots, h_{i}\right\}=\emptyset \\
\left(h_{1}, \ldots, h_{i}\right) \hookleftarrow_{i} t= & \left(\lambda x \cdot\left\langle\left(h_{1}, \ldots, h_{i}\right) \stackrel{\mathcal{C}}{\hookleftarrow}_{i} x\right\rangle_{i}\right) t
\end{aligned}
$$

The idea behind the first equality is to capture and destroy the current contexts with $\mathcal{L}_{i}$; when the throw $\hookleftarrow_{i}$ is performed, only empty contexts are pushed on the context of level $i+1$. In the second equation, the delimiter $\langle\cdot\rangle_{i}$ effectively pushes the current contexts up to level $i$ on the context of level $i+1$, and the throw $\stackrel{\mathcal{C}}{\leftrightarrow}$ i restores the captured contexts, discarding only the empty contexts $\bullet_{1}, \ldots, \bullet_{i}$ in the process. One can check that both equations are sound with respect to the CPS translation.

We can derive a typing rule for $\stackrel{\mathcal{C}}{\leftrightarrow}$ i from the type system of Figure 3 using the equation above, or directly from its CPS translation:

$$
\begin{gathered}
C_{1}=S \triangleright C_{2} \ldots \triangleright C_{n+1} \\
\Gamma ; \Delta \vdash_{n} h_{1}: C_{1} \quad \ldots \quad \Gamma ; \Delta \vdash_{n} h_{i}: C_{i} \\
D_{1}^{\prime}=S \triangleright C_{2}^{\prime} \triangleright \ldots C_{i}^{\prime} \triangleright C_{i+1} \triangleright \ldots C_{n+1} \\
\Gamma ; \Delta \vdash_{n} t: D_{1}^{\prime}, D_{2}, \ldots, D_{n+1} \\
\hline \Gamma ; \Delta \vdash_{n}\left(h_{1}, \ldots, h_{i}\right) \stackrel{\mathcal{C}}{{ }_{i}} t: D_{1}, D_{2}, \ldots, D_{n+1}
\end{gathered}
$$

As in the previous case, the properties of subject reduction, soundness w.r.t. the CPS translation, and termination of evaluation hold for the new system, therefore one can use interchangeably the two throw operators.

\section{MORE FLEXIBLE CONTROL OPERA- TORS}

In this section we consider some variants of the operators introduced in Section 2. Instead of capturing and throwing to continuous sequences of contexts starting from $1\left(E_{1}, \ldots, E_{i}\right)$, we allow capture and throw to any sequence of contexts $E_{i_{1}}, \ldots, E_{i_{j}}$, where $1 \leq i_{1}<i_{2}<\ldots<i_{j} \leq n$. The syntax of terms is now defined as follows:

$$
\begin{aligned}
t::= & x|\lambda x . t| t t\left|\mathcal{L}^{*}\left(k_{i_{1}}, \ldots, k_{i_{j}}\right) . t\right|\langle t\rangle_{i} \mid \\
& \left(h_{i_{1}}, \ldots, h_{i_{j}}\right) \stackrel{*}{\longleftarrow} t,
\end{aligned}
$$

the syntax of level-1 contexts is adjusted accordingly:

$$
E_{1}::=\bullet_{1}\left|v E_{1}\right| E_{1} t \mid\left(E_{i_{1}}, \ldots, E_{i_{j}}\right) \hookleftarrow_{i} E_{1}
$$

and the remaining syntactic categories are defined as before. The CPS translation, reduction rules, and typing rules for the operators $\mathcal{L}^{*}$ and $\stackrel{*}{\hookleftarrow}$ are summarized in Figure 5 .

Using the same proof techniques as in the previous section, we can also prove the following results.

Theorem 4 (Subject Reduction). If $\Gamma ; \Delta \vdash_{n} p: S$ and $p \rightarrow_{\mathrm{v}} p^{\prime}$ then $\Gamma ; \Delta \vdash_{n} p^{\prime}: S$.

Proposition 5 (SOUNDNESS OF TYPING WRT. CPS). The following implications hold:

$$
\begin{aligned}
& \text { 1. If } \Gamma ; \Delta \vdash_{n} t: C_{1}, \ldots, C_{n+1} \text {, then } \bar{\Gamma} ; \bar{\Delta} \vdash \bar{t}: \overline{C_{1}} \rightarrow \\
& \quad \ldots \rightarrow \overline{C_{n+1}} \rightarrow o \text {. } \\
& \text { 2. If } \Gamma ; \Delta \vdash_{n} E_{i}: C_{i} \text {, then } \bar{\Gamma} ; \bar{\Delta} \vdash \llbracket E_{i} \rrbracket: \overline{C_{i}} \text {, for all } \\
& i=1, \ldots, n \text {. }
\end{aligned}
$$

Proposition 6 (Soundness of Reduction WrT. CPS). If $p \rightarrow_{\mathrm{v}} p^{\prime}$, then $\bar{p}={ }_{\beta \eta} \overline{p^{\prime}}$.

Theorem 5 (Termination of evaluation). Let $t$ be a closed plain term such that $\cdot ; \cdot \vdash_{n} t: C_{1}, \ldots, C_{n+1}$ and $\mathcal{I}_{i}\left(C_{i}\right)$ hold for all $i=1, \ldots, n+1$. Then $\mathcal{N}\left(\left\langle t, \bullet_{1}, \ldots, \bullet_{n+1}\right\rangle\right)$ holds. 


\section{CPS translation}

$$
\begin{aligned}
& \overline{\mathcal{L}^{*}\left(k_{i_{1}}^{\prime}, \ldots, k_{i_{j}}^{\prime}\right) \cdot t}=\lambda k_{1} \ldots k_{n+1} . \bar{t}\left\{k_{i_{1}}^{\prime} / k_{i_{1}}, \ldots, k_{i_{j}}^{\prime} / k_{i_{j}}\right\} c_{1} \ldots c_{i_{j}} k_{i_{j}+1} \ldots k_{n+1} \\
& \text { where } c_{l}=\left\{\begin{array}{ll}
\theta_{l} & \text { if } j \in\left\{i_{1}, \ldots, i_{l}\right\} \\
k_{l} & \text { otherwise }
\end{array} \text { for all } 1 \leq l \leq i_{j}\right. \\
& \overline{\left(h_{i_{1}} \ldots h_{i_{j}}\right) \stackrel{*}{\leftarrow} t}=\lambda k_{1} \ldots k_{n+1} \cdot \bar{t}\left(\lambda v_{0} k_{2}^{\prime} \ldots k_{n+1}^{\prime} \cdot \llbracket h_{i_{1}} \rrbracket v_{0} d_{i_{1}+1} \ldots d_{i_{j}}\left(\lambda v_{1} k_{i_{j}+2}^{\prime \prime} \ldots k_{n+1}^{\prime \prime} \cdot k_{1} v_{1} k_{2}^{\prime} \ldots k_{i_{j}+1}^{\prime} k_{i_{j}+2}^{\prime \prime} \ldots k_{n+1}^{\prime \prime}\right)\right. \\
& \left.k_{i_{j}+2}^{\prime} \ldots k_{n+1}^{\prime}\right) k_{2} \ldots k_{n+1} \\
& \text { where } d_{l}=\left\{\begin{array}{ll}
\llbracket h_{l} \rrbracket & \text { if } j \in\left\{i_{1}, \ldots, i_{l}\right\} \\
\theta_{l} & \text { otherwise }
\end{array} \text { for all } i_{1} \leq l \leq i_{j}\right.
\end{aligned}
$$

\section{Reduction rules}

$$
\begin{aligned}
& (\text { capture } *) \quad\left\langle\mathcal{L}^{*}\left(k_{i_{1}}, \ldots, k_{i_{j}}\right) . t, E_{1}, \ldots, E_{n+1}\right\rangle \quad \rightarrow_{\mathrm{v}} \quad\left\langle t\left\{E_{1} / k_{i_{1}}, \ldots, E_{i_{j}} / k_{i_{j}}\right\}, E_{1}^{\prime}, \ldots, E_{i_{j}}^{\prime}, E_{i_{j}+1}, \ldots, E_{n+1}\right\rangle \\
& \text { where } E_{l}^{\prime}=\left\{\begin{array}{ll}
\bullet_{l} & \text { if } j \in\left\{i_{1}, \ldots, i_{l}\right\} \\
E_{l} & \text { otherwise }
\end{array} \text { for all } 1 \leq l \leq i_{j}\right. \\
& (\text { throw } *) \quad\left\langle\left(E_{i_{1}}^{\prime}, \ldots, E_{i_{j}}^{\prime}\right) \stackrel{*}{\longleftarrow} v, E_{1}, \ldots, E_{n+1}\right\rangle \quad \rightarrow_{\mathrm{v}} \quad\left\langle v, E_{1}^{\prime \prime}, \ldots, E_{i_{j}}^{\prime \prime}, E_{i_{j}+1} \cdot\left(E_{i_{j}} \ldots\left(E_{2} . E_{1}\right) \ldots\right), E_{i_{j}+2}, \ldots, E_{n+1}\right\rangle \\
& \text { where } E_{l}^{\prime \prime}=\left\{\begin{array}{ll}
E_{l}^{\prime} & \text { if } j \in\left\{i_{1}, \ldots, i_{l}\right\} \\
\bullet & \text { otherwise }
\end{array} \text { for all } i_{1} \leq l \leq i_{j}\right.
\end{aligned}
$$

\section{Typing rules}

$$
\begin{aligned}
& \frac{\mathcal{I}_{l}\left(D_{l}\right) \text { if } l \in\left\{i_{1}, \ldots, i_{j}\right\} \quad D_{l}=C_{l} \text { if } l \notin\left\{i_{1}, \ldots, i_{j}\right\} \quad \Gamma ; \Delta, k_{i_{1}}: C_{i_{1}} \ldots, k_{i_{j}}: C_{i_{j}} \vdash_{n} t: D_{1}, \ldots, D_{i_{j}}, C_{i_{j}+1} \ldots C_{n+1}}{\Gamma ; \Delta \vdash_{n} \mathcal{L}^{*}\left(k_{i_{1}}, \ldots, k_{i_{j}}\right) . t: C_{1}, C_{2}, \ldots, C_{n+1}} \\
& C_{i_{1}}=S \triangleright C_{i_{1}+1} \ldots \triangleright C_{n+1} \quad C_{i_{j}+1}=T \triangleright C_{i_{j}+2}^{\prime} \ldots \triangleright C_{n+1}^{\prime} \quad \mathcal{I}_{l}\left(C_{l}\right) \text { if } l \notin\left\{i_{1}, \ldots, i_{j}\right\} \text { and } i_{1} \leq l \leq i_{j} \\
& \Gamma ; \Delta \vdash_{n} h_{i_{1}}: C_{i_{1}} \quad \ldots \quad \Gamma ; \Delta \vdash_{n} h_{i_{j}}: C_{i_{j}} \\
& \Gamma ; \Delta \vdash_{n} t: S \triangleright C_{2}^{\prime \prime} \triangleright \ldots C_{i+1}^{\prime \prime} \triangleright C_{i+2} \triangleright \ldots C_{n+1}, D_{2}, \ldots, D_{n+1} \\
& \Gamma ; \Delta \vdash_{n}\left(h_{i_{1}}, \ldots, h_{i_{j}}\right) \hookleftarrow_{i} t: T \triangleright C_{2}^{\prime \prime} \triangleright \ldots C_{i_{j}+1}^{\prime \prime} \triangleright C_{i_{j}+2}^{\prime} \triangleright \ldots C_{n+1}^{\prime}, D_{2}, \ldots, D_{n+1}
\end{aligned}
$$

Figure 5: CPS translation, reduction rules, and typing rules for $\mathcal{L}^{*}$ and $\stackrel{*}{\hookleftarrow}$

Expressiveness. We first show how to simulate the operators $\mathcal{L}_{i}$ and $\stackrel{*}{\leftarrow}$ with $\mathcal{S}_{i}$ and $\stackrel{\mathcal{S}}{\leftarrow}_{i}$. We use the same translation as in Section 2.5, except that we translate $\stackrel{*}{\leftarrow}$ in the following way:

$$
\begin{aligned}
& \left(\left(h_{i_{1}}, \ldots, h_{i_{j}}\right) \stackrel{*}{\leftarrow} t\right)^{\circ}= \\
& \quad\left(\lambda x .\left\langle K_{i_{j}}{\stackrel{\stackrel{\mathcal{S}}{ }}{i_{j}}} \ldots\left\langle K_{2} \stackrel{\mathcal{S}}{\hookleftarrow}_{2}\left\langle K_{1} \stackrel{\mathcal{S}}{\hookleftarrow}_{1} x\right\rangle_{1}\right\rangle_{2} \ldots\right\rangle_{i_{j}}\right) t^{\circ}
\end{aligned}
$$

with $K_{l}=h_{l}^{\circ}$ if $l \in\left\{i_{1}, \ldots, i_{j}\right\}$ and $K_{l}=\left(\bullet_{1}, \ldots, \bullet_{l}\right)$ otherwise. In the translation, if $l \notin\left\{h_{i_{1}}, \ldots, h_{i_{j}}\right\}$, then we restore the empty context $\bullet l$ as the current context of level $l$ by throwing to $\bullet_{l}$ (in fact to the tuple $\left(\bullet_{1}, \ldots, \bullet_{l}\right.$ ), but only the last context matters). Otherwise, we throw to $h_{l}^{\circ}$, as in the translation for $\leftarrow_{i}$. Because the translation of $\mathcal{L}_{i}$ remains unchanged, we still throw to tuples of contexts captured by a singular shift, as required by the semantics of shift. Expressing $\mathcal{L}^{*}$ with $\mathcal{S}_{i}$ seems more difficult, because $\mathcal{L}^{*}$ may capture some contexts and leave the first one unchanged, while $\mathcal{S}_{i}$ always captures a tuple of contexts, starting from the first one. We conjecture that $\mathcal{L}^{*}$ cannot be expressed with $\mathcal{S}_{i}$.
As in Section 2.5, we may consider an alternative throw operator $\stackrel{\mathcal{C} *}{\hookleftarrow}$, such that $\left(E_{i_{1}}, \ldots, E_{i_{j}}\right) \stackrel{\mathcal{C} *}{\hookleftarrow} v$ replaces the current contexts at positions $i_{1}, \ldots, i_{j}$ by $E_{i_{1}}, \ldots, E_{i_{j}}$, and leaves the other ones unchanged. We define this operator via its CPS translation:

$$
\begin{aligned}
\overline{\left(h_{i_{1}}, \ldots, h_{i_{j}}\right)} \stackrel{\stackrel{\mathcal{C} *}{\hookleftarrow} t}{\leftrightarrows}=\lambda k_{1} \ldots k_{n+1} . \\
\bar{t}\left(\lambda v_{0} k_{2}^{\prime} \ldots k_{n+1}^{\prime} . c_{1} v_{0} c_{2} \ldots c_{i_{j}} k_{i_{j}+1}^{\prime} \ldots k_{n+1}^{\prime}\right) \\
k_{2} \ldots k_{n+1}
\end{aligned}
$$

where $c_{l}=\llbracket h_{l} \rrbracket$ if $l \in\left\{i_{1}, \ldots, i_{j}\right\}$ and $c_{l}=k_{l}$ otherwise. The corresponding reduction rule is:

$$
\begin{aligned}
\left\langle\left(E_{1}^{\prime}, \ldots, E_{i}^{\prime}\right) \stackrel{\mathcal{C} *}{\hookleftarrow} v, E_{1}, \ldots, E_{n+1}\right\rangle \rightarrow_{\mathrm{v}} \\
\left\langle v, E_{1}^{\prime \prime}, \ldots, E_{i_{j}}^{\prime \prime}, E_{i_{j}+1}, \ldots, E_{n+1}\right\rangle
\end{aligned}
$$

where $E_{l}^{\prime \prime}=E_{l}^{\prime}$ if $l \in\left\{i_{1}, \ldots, i_{j}\right\}$ and $E_{l}^{\prime \prime}=E_{l}$ otherwise. We can express $\stackrel{*}{\leftarrow}$ with $\stackrel{\mathcal{C} *}{\leftarrow}$ as follows:

$$
\left(h_{i_{1}}, \ldots, h_{i_{j}}\right) \stackrel{*}{\hookleftarrow} t=\left(\lambda x .\left\langle\left(h_{i_{1}}, \ldots, h_{i_{j}}\right) \stackrel{\mathcal{C} *}{\hookleftarrow} x\right\rangle_{i_{j}}\right) t
$$

Roughly, the delimiter $\langle\cdot\rangle_{i_{j}}$ pushes the current contexts on 
$E_{i_{j}+1}$, and the captured contexts are then restored with $\stackrel{\mathcal{C} *}{\hookleftarrow}$. However, simulating $\stackrel{\mathcal{C} *}{\leftarrow}$ with $\stackrel{*}{\leftarrow}$ seems difficult, mainly because $\left(E_{i_{1}}, \ldots, E_{i_{j}}\right) \stackrel{\mathcal{C} *}{\hookleftarrow} v$ leaves contexts $E_{l}$ such that $l \leq$ $i_{j}$ and $l \notin\left\{i_{1}, \ldots, i_{j}\right\}$ unchanged, while $\left(E_{i_{1}}, \ldots, E_{i_{j}}\right) \stackrel{*}{\leftarrow} v$ replaces them with $\bullet_{1}$. We conjecture that $\stackrel{\mathcal{C} *}{\hookleftarrow}$ cannot be expressed with $\stackrel{*}{\longleftarrow}$.

\section{CONCLUSION AND PERSPECTIVES}

We have developed the most expressive monomorphic type system for a family of control operators in the CPS hierarchy and for this type system we have proved subject reduction, soundness with respect to CPS, and termination of evaluation. We believe that the present article, as a sequel to the operational foundations of the CPS hierarchy built by the first two authors and Danvy, is another step towards better understanding of the CPS hierarchy, and that it can lead to new applications, implementations as well as theoretical results on this topic.

There are several directions for future research related to the present work. First of all, as opposed to the type systems of Danvy and Yang [9] and of Murthy [22] the type system presented in this work allows for computations that modify the answer type of continuations at an arbitrary level of the hierarchy, which should open new possibilities for practical applications that otherwise could only be expressed in an untyped setting.

Building an experimental implementation of the hierarchy with types à la Danvy and Filinski as presented in this article is another task. In particular, one can use the syntactic correspondence between context-based reduction semantics and abstract machines [6] to obtain an abstract machine equivalent with the reduction semantics of this article, or one could adjust the existing abstract machine for the hierarchy [5] accordingly and prove its correctness with respect to the reduction semantics. Devising a type reconstruction algorithm for the hierarchy should not pose any serious problems. A more ambitious goal is to marry the type system from this work with ML-polymorphism, which could be done along the lines presented by Asai and Kameyama [2].

It would be interesting to formalize the proof of termination of evaluation in the CPS hierarchy in a logical framework such as the Calculus of Inductive Constructions of Coq. As has been shown before [3, 4], normalization proofs by Tait's context-based method yield, through program extraction from proofs, non-trivial evaluators in CPS and the program extraction mechanism of Coq could be helpful for this task.

The present article focuses on the CPS hierarchy under the call-by-value reduction strategy. A natural next step is to see how the type system à la Danvy and Filinski for call-byname shift and reset introduced by the first two authors [4] generalizes to a call-by-name hierarchy. It would be instructive to relate such a hierarchy to the one recently presented by Saurin [24].

Finally, the still open question of the logical interpretation of delimited continuations through the Curry-Howard iso- morphism carries over from shift and reset to the hierarchy.

\section{REFERENCES}

[1] Z. M. Ariola, H. Herbelin, and A. Sabry. A proof-theoretic foundation of abortive continuations. Higher-Order and Symbolic Computation, 20(4):403-429, 2007.

[2] K. Asai and Y. Kameyama. Polymorphic delimited continuations. In Proceedings of the Fifth Asian symposium on Programming Languages and Systems, $A P L A S^{\prime} 07$, number 4807 in Lecture Notes in Computer Science, pages 239-254, Singapore, Dec. 2007.

[3] M. Biernacka and D. Biernacki. A context-based approach to proving termination of evaluation. In Proceedings of the 25th Annual Conference on Mathematical Foundations of Programming Semantics(MFPS XXV), Oxford, UK, Apr. 2009.

[4] M. Biernacka and D. Biernacki. Context-based proofs of termination for typed delimited-control operators. In F. J. López-Fraguas, editor, Proceedings of the 11th $A C M-S I G P L A N$ International Conference on Principles and Practice of Declarative Programming (PPDP'09), Coimbra, Portugal, Sept. 2009. ACM Press.

[5] M. Biernacka, D. Biernacki, and O. Danvy. An operational foundation for delimited continuations in the CPS hierarchy. Logical Methods in Computer Science, 1(2:5):1-39, Nov. 2005. A preliminary version was presented at the Fourth ACM SIGPLAN Workshop on Continuations (CW'04).

[6] M. Biernacka and O. Danvy. A syntactic correspondence between context-sensitive calculi and abstract machines. Theoretical Computer Science, 375(1-3):76-108, 2007.

[7] O. Danvy and A. Filinski. A functional abstraction of typed contexts. DIKU Rapport 89/12, DIKU, Computer Science Department, University of Copenhagen, Copenhagen, Denmark, July 1989.

[8] O. Danvy and A. Filinski. Abstracting control. In M. Wand, editor, Proceedings of the 1990 ACM Conference on Lisp and Functional Programming, pages 151-160, Nice, France, June 1990. ACM Press.

[9] O. Danvy and Z. Yang. An operational investigation of the CPS hierarchy. In S. D. Swierstra, editor, Proceedings of the Eighth European Symposium on Programming, number 1576 in Lecture Notes in Computer Science, pages 224-242, Amsterdam, The Netherlands, Mar. 1999. Springer-Verlag.

[10] A. Filinski. Representing monads. In H.-J. Boehm, editor, Proceedings of the Twenty-First Annual ACM Symposium on Principles of Programming Languages, pages 446-457, Portland, Oregon, Jan. 1994. ACM Press.

[11] A. Filinski. Representing layered monads. In A. Aiken, editor, Proceedings of the Twenty-Sixth Annual ACM Symposium on Principles of Programming Languages, pages 175-188, San Antonio, Texas, Jan. 1999. ACM Press.

[12] R. Harper, B. F. Duba, and D. MacQueen. Typing first-class continuations in ML. Journal of Functional Programming, 3(4):465-484, Oct. 1993. A preliminary 
version was presented at the Eighteenth Annual ACM Symposium on Principles of Programming Languages (POPL 1991).

[13] H. Herbelin and S. Ghilezan. An approach to call-by-name delimited continuations. In P. Wadler, editor, Proceedings of the Thirty-Fifth Annual ACM Symposium on Principles of Programming Languages, pages 383-394. ACM Press, Jan. 2008.

[14] Y. Kameyama. Axioms for delimited continuations in the CPS hierarchy. In J. Marcinkowski and A. Tarlecki, editors, Computer Science Logic, 18th International Workshop, CSL 2004, 13th Annual Conference of the EACSL, Proceedings, volume 3210 of Lecture Notes in Computer Science, pages 442-457, Karpacz, Poland, Sept. 2004. Springer.

[15] Y. Kameyama, O. Kiselyov, and C. Shan. Shifting the stage: Staging with delimited control. In G. Puebla and G. Vidal, editors, Proceedings of the 2009 ACM SIGPLAN Symposium on Partial Evaluation and Semantics-Based Program Manipulation (PEPM 2009), pages 111-120, Savannah, GA, Jan. 2009. ACM Press.

[16] O. Kiselyov. Call-by-name linguistic side effects. In Proceedings of the 2008 Workshop on Symmetric Calculi and Ludics for the Semantic Interpretation, Hamburg, Germany, Aug. 2008.

[17] O. Kiselyov. Delimited control in ocaml, abstractly and concretely: System description. In M. Blume and G. Vidal, editors, Functional and Logic Programming, 10th International Symposium, FLOPS 2010, number 6009 in Lecture Notes in Computer Science, pages 304-320, Sendai, Japan, Apr. 2010. Springer.

[18] O. Kiselyov and Chung-chieh. Embedded probabilistic programming. In W. Taha, editor, Domain-Specific Languages, DSL 2009, number 5658 in Lecture Notes in Computer Science, pages 360-384, Oxford, UK, July 2009. Springer.

[19] O. Kiselyov and C. Shan. Delimited continuations in operating systems. In B. Kokinov, D. C. Richardson, T. R. Roth-Berghofer, and L. Vieu, editors, Modeling and Using Context, 6th International and Interdisciplinary Conference, CONTEXT 200\%, number 4635 in Lecture Notes in Artificial Intelligence, pages 291-302, Roskilde, Denmark, Aug. 2007. Springer.

[20] O. Kiselyov and C. Shan. A substructural type system for delimited continuations. In S. R. D. Rocca, editor, Typed Lambda Calculi and Applications, 8th International Conference, TLCA 200\%, number 4583 in Lecture Notes in Computer Science, pages 223-239, Paris, France, June 2007. Springer-Verlag.

[21] M. Masuko and K. Asai. Direct implementation of shift and reset in the MinCaml compiler. In A. Rossberg, editor, Proceedings of the ACM SIGPLAN Workshop on $M L, M L ' 09$, pages 49-60, Edinburgh, UK, Aug. 2009.

[22] C. R. Murthy. Control operators, hierarchies, and pseudo-classical type systems: A-translation at work. In O. Danvy and C. L. Talcott, editors, Proceedings of the First ACM SIGPLAN Workshop on Continuations (CW'92), Technical report STAN-CS-92-1426, Stanford University, pages 49-72, San Francisco,
California, June 1992.

[23] G. D. Plotkin. Call-by-name, call-by-value and the $\lambda$-calculus. Theoretical Computer Science, 1:125-159, 1975.

[24] A. Saurin. A hierarchy for delimited continuations in call-by-name. In L. Ong, editor, Foundations of Software Science and Computation Structures, 13th International Conference, FOSSACS 2010, number 6014 in Lecture Notes in Computer Science, pages 374-388, Paphos, Cyprus, Mar. 2010. Springer-Verlag.

[25] W. W. Tait. Intensional interpretation of functionals of finite type I. Journal of Symbolic Logic, 32:198-212, 1967.

[26] N. Zeilberger. Polarity and the logic of delimited continuations. In J.-P. Jouannaud, editor, Proceedings of the 25th IEEE Symposium on Logic in Computer Science (LICS 2010), pages 219-227, Edinburgh, UK, July 2010. IEEE Computer Society Press. 